\title{
Redecir lo humano
}

Resaying the Human

Redizer o humano

\section{Juan Carlos Aguirre García*}

Artículo de investigación

Revista Colombiana de Educación, N. 72. Primer semestre de 2017, Bogotá, Colombia.

Para citar: Aguirre, J. C. (2017). Redecir lo humano. Revista Colombiana de Educación, (72), 177-197.

Fecha de recepción: 28/07/2011

Fecha de aceptación: 9/11/2016

Licenciado en Filosofia (Universidad Santo Tomás, Bogotá), Magíster en Filosofía (Universidad de Caldas, Manizales), Doctor (c) en Filosofía (Universidad Pontificia Bolivariana, Medellín). Profesor titular de la Universidad del Cauca, Colombia. correo electrónico: jcaguirre@anicauca.edu.co 


\section{Resumen}

Este artículo explora el fenómeno de la crisis de las humanidades a partir de algunas ideas del filósofo Emmanuel Levinas. En vez de centrarse en las discusiones sobre los ataques de los que son objeto las humanidades, sobre todo en épocas recientes, el análisis se dirige hacia la crisis del sentido de las humanidades como causa primordial de la crisis de las humanidades. El artículo comienza describiendo el carácter oscuro del hombre y lo humano en la contemporaneidad, continúa con la presentación de la relación que se ha tejido entre ciencias naturales y humanidades desde la modernidad, y culmina con dos apartados en los que se plantea la alternativa levinasiana. El propósito central es sostener que el sentido de las humanidades no depende tanto de la comprensión del sujeto o las expresiones culturales, sino de la respuesta que pueda darse al llamado del Otro, lo que exige nuevos modos de decir lo humano.

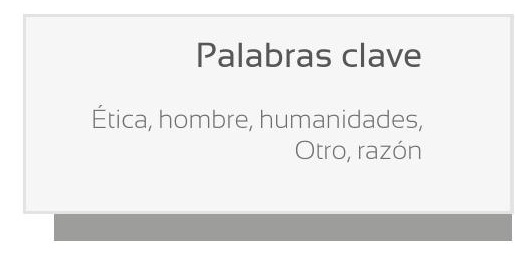
Abstract
This paper explores the phenomenon of the crisis of the humanities ba-
sed on some ideas of philosopher Emmanuel Levinas. Rather than fo-
cusing on discussions of recent attacks on the humanities, the analysis
addresses the crisis of the sense of the humanities as the main cause of
its crisis. First, the paper describes the dark nature of humankind and the
human in the contemporaneity. Then, it shows the relationship that has
been emerging, since modernity, between natural sciences and humani-
ties. Finally, it devotes two sections to discuss the Levinasian alternative.
The thesis supported here is that the sense of the humanities does not
depend on the understanding of the Subject or his/her cultural expres-
sions, but rather on the response to the Other's call, which demands new
ways to explain the human.

\section{Resumo}

Este artigo explora o fenómeno da crise das humanidades a partir de algumas ideias do filosofo Emmanuel Levinas. Em lugar de centrar-se nas discussões sobre os ataques dos que as humanidades são objeto, especialmente em épocas recentes, a analise está focalizada sobre a crise do sentido das humanidades como causa primordial da crise das humanidades. O artigo inicia com uma descrição do caráter escuro do homem e o humano na contemporaneidade, continua com a apresentação da relação tecida entre as ciências naturais e humanidades desde a modernidade, e finaliza com duas seções nas que se estabelece a alternativa levinasiana. O proposito central é sustentar que o sentido das humanidades não depende principalmente da compreensão do sujeito ou das expressões culturais, mas da resposta que é possível obter ao chamado do Outro, o que exige novos modos de dizer o humano.

\section{Palavras chave}

Ética, homem, humanidades, Outro, razão 


\section{Introducción}

Pareciera que la frase con la que Foucault cierra su ya clásico libro Las palabras y las cosas (1966/2001), escrita a modo de presentimiento o apuesta, hubiera alcanzado su cumplimiento. El presagio foucaultiano según el cual "el hombre se borraría, como en los límites del mar un rostro de arena" (Foucault, 2001, p. 375) ha dejado de ser tal y se ha convertido en una realidad cruda. Son abundantes los diagnósticos de la célebre tesis de la desaparición del hombre ${ }^{1}$, cada uno de ellos inscrito en diferentes horizontes; basta citar un par de ellos:

En 2010, la filósofa Martha C. Nussbaum publicó un corto ensayo titulado Sin fines de lucro. Por qué la democracia necesita de las humanidades, que bien podría tomarse como un manifiesto en pro de las humanidades. En este texto, Nussbaum habla de una "crisis silenciosa" de "proporciones gigantescas y de enorme gravedad a nivel mundial" (2010, p. 19). La alarma que enciende la autora se relaciona con "la crisis mundial en materia de educación" (p. 20) y cómo esta crisis pone en riesgo el futuro de la democracia; de ahí su alcance mundial. El argumento de la filósofa plantea que se está privilegiando una educación que contribuya de manera inmediata al crecimiento económico y a la rentabilidad; dicho privilegio va en desmedro de asignaturas que han ido tomando tintes de "ornamentos inútiles" (p. 20), especialmente las artes y las humanidades. Sin embargo, continúa Nussbaum, estas asignaturas despliegan ciertas capacidades en los seres humanos; por ejemplo: el pensamiento crítico, y la capacidad de vivir con otros como "ciudadanos del mundo" y de imaginar con compasión las dificultades del prójimo (2010, p. 26). Tales capacidades son esenciales a la democracia. En consecuencia, la supresión de las artes y las humanidades del currículo escolar es una amenaza al futuro de la democracia.

El otro diagnóstico, si bien desarraigado del suelo educativo y afincado más bien en la reflexión especulativa, también parte de la premisa de que el humanismo, en general, y las humanidades, en particular, están en crisis; ya no en razón de sus fines, sino de su objeto. Se trata del trabajo de Cary Wolfe, What is posthumanism? (2010), en el que resuenan frecuentemente Foucault y los posmodernos franceses. Allí plantea que el propósito del poshumanismo no es sobrepasar o rechazar al humano, sino describirlo con sus modos característicos de comunicación,

1 Aun cuando célebre, esta tesis de Foucault puede llevar a malentendidos. Un trabajo esclarecedor a este respecto es el artículo de Castro (2005), donde se plantea la conjetura de que "el desenlace de Las palabras y las cosas no se reduce a la dimensión negativa que parece contener la expresión 'muerte del hombre' sino que, al contrario, apunta a lo que representaría la pars construens de la filosofía de Foucault". El presente artículo está en consonancia con esta conjetura de Castro.

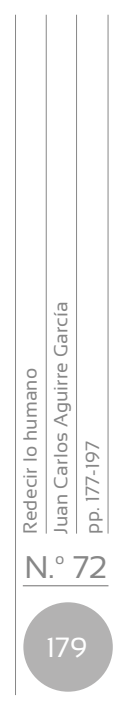


interacción, significaciones sociales y valores afectivos con mayor especificidad, una vez se haya removido el sentido del dominio ontológicamente cerrado de la conciencia, la razón, la reflexión, etc. (Wolfe, 2010, p. xxv). Según algunos postulados posmodernos, el poshumanismo forzaría a repensar lo humano, en la medida en que este se ha asumido como Homo sapiens, para recontextualizarlo en términos de su relación con otros seres vivos, con sus propias maneras de hacer mundo $y$, sobre todo, para describirlo como un ser protésico que ha coevolucionado con varias formas de tecnicidad y materialidad, "formas que son radicalmente 'no humanas' $y$, sin embargo, hacen que el humano sea lo que es" (Wolf, 2010, p. xxv).

Estos dos diagnósticos dan cuenta de que, en efecto, las humanidades están en vilo, no solo porque "cada vez son más minimizadas, invisibilizadas y eliminadas de los planes de estudio tanto en Colombia como en el mundo" (Vargas y Prada, 2016), sino también porque parece haber una nueva concepción de humano -nueva con relación a la reflexión moderna- a la que deben hacer frente las humanidades de hoy. Este artículo se ubica en esta segunda línea reflexiva, esto es, explora la crisis de las humanidades en razón de su objeto: lo humano. Por ser multidimensional, tal objeto se abordará sin fines exhaustivos; más bien, se centrará en la obsolescencia del lenguaje de las humanidades que hacen el juego a una racionalidad unívoca, y explorará las posibilidades que abre un discurso que responda primeramente al otro, en vez de obsesionarse con la pretensión de validez. Si bien se opta por la segunda línea reflexiva, el artículo concluye mencionando algunas implicaciones que esto tiene para la formación posgradual en humanidades.

\section{Las humanidades como mímesis de las ciencias "duras"2}

Contrario a lo que puede pensarse, las humanidades no gozan de un linaje que se retrotraiga más allá de la revolución científica del siglo xvı; en consecuencia, no gozan de una pretendida autoridad otorgada por una primogenitura. Si bien puede hacerse un rastreo del término humanitas

2 Aunque esta distinción entre humanidades y ciencias duras (sin comillas) parece obsoleta, es curioso notar cómo algunos periodistas la mantienen en la actualidad, incluso cuando intentan superarla. Esta frase es un ejemplo de ello: "hace falta derrumbar ese muro entre las ciencias duras y las humanidades en varios países del mundo y, particularmente, en Colombia. El reto está en lograr construir un país donde sean valorados tanto los profesores de poesía, como los biólogos marinos" ("¿Quién piensa en las artes y las humanidades?", 2016). En este apartado se ha matizado la palabra duras, toda vez que la distinción parece insostenible en los términos en que tradicionalmente se ha dado. A este respecto ver el artículo de la epistemóloga Susan Haack (2002). 
y dotarlo de sentido original, el grupo de disciplinas que hoy conforman las humanidades (o, para ser más precisos, las ciencias humanas) es posterior a las ciencias de la naturaleza; por tanto, las humanidades son posteriores a las ciencias naturales. Afirmar lo contrario sería creer, ingenuamente, que la ciencia surgida en la modernidad es la misma que la ciencia previa, por ejemplo, la aristotélica; o sostener que la ciencia moderna es una etapa más en el proceso evolutivo de la ciencia (¡Como si no hubiéramos padecido el escándalo de Kuhn!).

Desde distintas tradiciones, algunos pensadores han respaldado esta idea. Foucault, en la ya mencionada obra, afirma:

La primera cosa que ha de comprobarse es que las ciencias humanas no han recibido como herencia un cierto dominio ya dibujado, medido quizá en su conjunto, pero que se ha dejado sin cultivo, y que tendrían la tarea de trabajar con conceptos científicos al fin y con métodos positivos; el siglo xvIII no les ha transmitido bajo el nombre de hombre o de naturaleza humana un espacio circunscrito desde el exterior pero aún vacío, que tendrían el deber de cubrir y analizar en seguida. El campo epistemológico que recorren las ciencias humanas no ha sido prescrito de antemano [...]; y las ciencias humanas no aparecieron hasta que, bajo el efecto de algún racionalismo presionante, de algún problema científico no resuelto, de algún interés práctico, se decidió hacer pasar al hombre [...] al lado de los objetos científicos. (2001, p. 334).

En similar sentido, en el clásico Informe de la Comisión Gulbenkian para la reestructuración de las ciencias sociales, coordinado por Wallerstein (2006), puede leerse lo siguiente:

La ciencia, es decir la ciencia natural, estaba mucho más claramente definida que su alternativa, para la cual el mundo nunca se ha puesto de acuerdo en un nombre único. A veces Ilamada las artes, a veces las humanidades, a veces las letras o las bellas letras, a veces la filosofía y a veces incluso la cultura, o en alemán Geisteswissenschaften, la alternativa de "la ciencia" ha tenido un rostro y un énfasis variables, una falta de coherencia interna que no ayudó a sus practicantes a defender su caso ante las autoridades, especialmente debido a su aparente incapacidad de presentar resultados "prácticos". Porque había empezado a estar claro que la lucha epistemológica sobre qué era conocimiento legítimo ya no era solamente una lucha sobre quién controlaría el conocimiento sobre la naturaleza (para el siglo xvIII estaba claro que los científicos naturales habían ganado los derechos exclusivos sobre ese campo) sino sobre quién controlaría el conocimiento sobre el mundo humano. (Wallerstein, 2006, p. 8). 
A partir de los textos citados, puede respaldarse la tesis de que las humanidades se configuraron con referencia a las ciencias naturales; en otras palabras, que las humanidades imitaron a las ciencias naturales. En primer lugar, si bien es cierto que había clasificaciones de los saberes antes de la revolución científica (véase, por ejemplo, la clasificación de las artes liberales en trivium y quadrivium), estas clasificaciones se rompieron con el advenimiento de la ciencia natural ${ }^{3}$. De ahí la interesante digresión de Wallerstein cuando en la cita unifica el universo de los saberes en la palabra ciencia, homologándola a "ciencia natural"; esto es, no es necesario señalar a qué hacemos mención cuando usamos la palabra ciencia pues esta nos remite, inmediatamente, a ciencia natural. Todo lo que se pretenda científico quedará reglamentado por los principios y métodos de este nuevo modo de pensar.

En segundo lugar, esta reconfiguración del saber, marcada por el privilegio de la ciencia natural, altera las concepciones previas de hombre y humano; es por ello que Foucault habla de un no dominio de las humanidades en este periodo, toda vez que la (nueva) ciencia y la técnica, que surgen en tándem, alteran la concepción de hombre. Con relación a la técnica, Blumenberg (2013) señala el siguiente aspecto:

Nuestra tradición está dominada, en gran medida, por la idea de que la naturaleza constituye la trabazón de un orden establecido a causa del hombre y orientado hacia el hombre. Es fácil ver cómo, en el marco de esta representación, las habilidades y prestaciones técnicas del ser humano solo podían desempeñar una función complementaria, que coadyuva a la naturaleza y ejecuta su finalidad [...]. El estadio moderno de la historia de la tecnicidad humana puede ser considerado, por ello, no solo desde el punto de vista del incremento cuantitativo de las prestaciones de los recursos de índole técnica. Más bien, detrás del crecimiento acelerado del ámbito de lo tecnológico hay una voluntad, conscientemente enfrentada con la realidad enajenada, de forzar mediante la técnica un nuevo carácter humano de la realidad. El ser humano reflexiona sobre las carencias de la naturaleza y su propia indigencia viéndolas como un acicate de todo su comportamiento. (pp. 36-37, énfasis en el original).

De acuerdo con esto, ante los nuevos acontecimientos, por ejemplo la técnica, la reflexión previa acerca de lo humano se queda corta (por no decir muda) y, en consecuencia, debe adoptar una nueva perspectiva acorde con las circunstancias apremiantes en las que el hombre se halla sumergido. Es claro, como sostiene Blumenberg para el caso

3 Más aún, en esta época se da una "rehabilitación de las 'artes mecánicas'” (Blumenberg, 2013, p. 65), es decir, al ámbito de los saberes se integran los "oficios", que justamente eran desdeñados por las artes liberales, volviéndose ahora relevantes para la consolidación de la (nueva) ciencia. 
de la técnica, que no se trata de que en la modernidad se haya dado un aumento de objetos; se trata de que en esta interacción con los objetos acaece un cambio sustancial de lo humano. Para tomar solo una de las palabras ya citadas de Wolf (2010), lo humano adopta ahora un carácter "protésico". Por ende, las humanidades actúan con retraso; el ritmo lo marca la innovación propuesta por los saberes recientemente entronizados.

En tercer lugar, pareciera, como consecuencia lógica de lo anterior, que si la ciencia y la técnica modernas exigen una nueva consideración de hombre, los discursos previos carecen de pertinencia, toda vez que respondían a situaciones ya superadas. Ahora bien, aparejada con esta idea, se encuentra que las humanidades operarían como una reflexión de segundo orden, esto es: meditarían sobre las nuevas configuraciones de lo humano, las cuales surgen de circunstancias sociales, políticas, económicas, científicas, técnicas y demás. Un cambio en tales circunstancias implicaría un deshacer y rehacer discursivo de las humanidades. Dichos discursos tendrían que trabajar, inexorablemente, "con conceptos científicos al fin y con métodos positivos" (Foucault, 2001, p. 334).

Un caso ilustrativo de estas ideas lo constituyen los trabajos de la profesora Eva Illouz. En su planteamiento del "Surgimiento del Homo Sentimentalis", la autora expone el lugar de las emociones en los discursos de la modernidad y cómo las teorizaciones acerca de las emociones coadyuvaron en la consolidación del proyecto moderno. Solo quisiera, en aras de sustentar las ideas del párrafo anterior, comentar brevemente su presentación sobre la imbricación entre psicología, administración e ingeniería. Para Illouz (2007), "en ninguna área esa influencia [de la psicología] fue tan palpable como en la empresa estadounidense, donde los psicólogos entrelazaron las emociones con el ámbito de la acción económica en una forma totalmente nueva de concebir la producción" (p. 32). Según la autora, a comienzos del siglo xx, "paralelamente a la retórica de los ingenieros [...] surgió otro discurso, impulsado por los psicólogos, que prestaban mucha atención al individuo, a la dimensión irracional de las relaciones de trabajo, así como a las emociones de los trabajadores" (IIlouz, 2007, pp. 34-35). La autora se interesa, fundamentalmente, por lo que denomina el "ethos comunicativo" en la empresa, en cómo la comunicación se vuelve el vehículo para expresar asertivamente los sentimientos de los empleados, cómo un buen gerente debe escuchar a sus empleados y cómo generar un clima de interacción en una empresa que, si bien es jerárquica y rígida, está inmersa en un contexto democrático. Concluye Illouz: "Así, el concepto y la práctica de la comunicación, que en un primer momento se presentó como técnica y como definición ideal del yo, se aplica ahora hasta para caracterizar a la empresa ideal" (2007, p. 56). 
De este caso se pueden extraer las siguientes ideas: (1) Si bien la reflexión humanista había abordado el asunto de las emociones desde hacía bastantes siglos, no se había postulado algo así como un Homo sentimentalis, con las implicaciones que esto tiene en el contexto capitalista; (2) la nueva reflexión sobre las emociones solo adquiere relevancia en la medida en que permite decir algo acerca de algo; (3) este decir algo exige que sea dicho en los mismos términos del algo al que se refiere o, por lo menos, en términos que contribuyan a que este se perfeccione; esto implica que el decir se especialice en una o varias áreas; (4) las humanidades interesan en el mundo "real" (en el caso considerado por Illouz, el mundo empresarial), solo si dicen algo directamente pertinente para tal mundo; (5) el Homo sentimentalis surge, como tal, solo en esta época ${ }^{4}$.

Afirmar, entonces, que las humanidades surgieron como mímesis de las ciencias duras, más que repetir un aparente lugar común, implica reconocer que su estatus como disciplinas se adquiere con referencia a las ciencias naturales; además, que el hombre o lo humano, su objeto de estudio, tiene que constituirse no autónomamente, sino en relación con los procesos históricos, económicos, políticos, científicos, técnicos. Finalmente, lo que demuestra una reflexión sobre la modernidad no es tanto que las humanidades, en la distribución de la hegemonía de los saberes, se ubicaron por debajo de las ciencias (naturales), sino que no hay algo que podamos Ilamar sustancial en lo humano; es decir, su naturaleza -si es que hay tal cosa-, es contingente ${ }^{5}$. Pretender prescribir qué es lo auténticamente humano en el hombre, sin recurrir a las circunstancias que lo determinan en una determinada época, sería alimentar una quimera.

4 No se afirma con esto que antes de esta época no hay sentimientos en el hombre, lo que sería un contrasentido. Lo que se afirma es que la categoría Homo sentimentalis -con las connotaciones que quiere darle Illouz, no solo en esta sino en muchas de sus investigaciones, por ejemplo las dedicadas al amor en la contemporaneidad (Illouz, 2012)-, adquiere un nuevo sentido en las circunstancias presentes de la sociedad y del hombre en ella. Por tal motivo, si se insiste en actualizar las teorías sobre la emoción, antes de la modernidad, tal actualización tendrá sentido, especialmente, si contribuye a la comprensión de esta dimensión del hombre contemporáneo.

5 A este respecto, la profesora Paula Sibilia expresa: "Una de las características que mejor definen al hombre es, precisamente, su indefinición: la proverbial plasticidad del ser humano" (2005, p. 10). Hago referencia a esta autora por sus rigurosos trabajos para repensar el ser humano en la época contemporánea y sus valiosos aportes para las humanidades por redecir. 


\section{La crisis de las humanidades a partir de la crisis de la disolución de su objeto}

Como se sugirió en el apartado introductorio, las humanidades están en crisis. Tal vez esta crisis está sobre diagnosticada; basta mencionar, al azar, algunos títulos interesantes al respecto, encontrados, en su mayoría, en columnas de opinión: "La crisis de las humanidades" (Delbanco, 2013), "The Real Humanities Crisis" (Gutting, 2013), "La crisis de las humanidades" (Cuartango, 2014), "Las humanidades, cada vez más cerca de su fin: el sTem acabará muy pronto con ellas" (Jiménez, 2014), "It's the End of the Humanities as We Know It. And I Feel Fine" (Greteman, 2014), "Humanities crisis? What crisis?" (Reisz, 2015). Los ejemplos podrían darse hasta la saciedad, lo mismo que las causas que se atribuyen, las concepciones de humanidades que se traslucen y las versiones apocalípticas u optimistas que se transmiten.

Por cuanto el interés de este artículo está en plantear que una de las causantes de la crisis de las humanidades podría buscarse en los discursos que se gestaron como respuesta al éxito de la ciencia moderna, discursos que abordaron al hombre "al lado de los objetos científicos" (Foucault, 2001, p. 334), y que, por tanto, una posible alternativa a tal discurso estaría en redecir lo humano, recurriré a las tesis del filósofo Emmanuel Levinas, especialmente las consignadas en su libro Humanismo del otro hombre (1972), para, en primer lugar, mostrar brevemente el modo como comprende tal crisis y, en el apartado siguiente, para evaluar su tesis en torno a lo que he llamado: redecir lo humano ${ }^{6}$. No considero que las ideas de este autor sean exclusivas o las definitivas (bien puede tomarse a Arendt, o a Nussbaum, o a otro pensador más citado a este respecto); el revisar sus tesis solo tiene la pretensión de introducir un nuevo contertulio y mostrar otros elementos de análisis y otra ruta para avizorar una salida plausible a la crisis de las humanidades.

Desde el comienzo, Levinas señala un aspecto que tal vez se haya pasado por alto en el análisis del primer apartado: al inicio se hizo alusión al lugar común según el cual las humanidades son posteriores a las ciencias naturales. Esta dualidad se puede percibir en gran parte de las columnas de opinión mencionadas. No obstante, Levinas ve a unas y otras como caras de la misma moneda; esto es, tanto las humanidades como las ciencias naturales forman parte de un mismo proyecto:

6 No pretendo ser original con esta expresión (que, a su vez, da título a este artículo); la misma puede encontrarse en el valioso trabajo del profesor Carl Cederberg, titulado: Resaying the Human. Levinas Beyond Humanism and Antihumanism (2010). 
Por el papel que le toca desempeñar, el sujeto pertenece, de hecho, a la gesta del ser y, a este título, se manifiesta a su vez: a sí mismo y a las ciencias humanas. No hay vida alguna que signifique fuera de esta verdad a la que sirve y en la que se muestra. El resto de lo humano le es ajeno. (Levinas, 1998, p. 14).

Debe aclararse que Levinas no confunde ingenuamente las manifestaciones de ambos discursos; no obstante, identifica un suelo común en el que confluyen realidad e inteligibilidad, trenzando relaciones de significación que no son más que una "totalidad iluminadora y necesaria para la propia percepción” (Levinas, 1998, p. 25). En el apartado siguiente se ampliará esta idea.

Es interesante ver cómo en el artículo "Humanismo y anarquía", escrito en 1968, Levinas ya habla de una "crisis del humanismo en nuestra época" (1998, p. 63). Esto es, la crisis ha sido diagnosticada hace, por lo menos, cinco décadas; solo que hasta ahora se siente con más fuerza en los ámbitos universitarios. La raíz de tal crisis se encuentra, a decir del autor, en "la experiencia de la ineficacia humana que revelan la abundancia misma de nuestros medios prácticos y la amplitud de nuestras ambiciones" (1998, p. 63). En consonancia con otros autores", Levinas plantea la crisis de la siguiente manera:

En el mundo en el que las cosas tienen su cometido, en el que los ojos, las manos y los pies saben encontrarlas, en el que la ciencia prolonga la topografía de la percepción y la praxis, aun cuando transfigure su espacio; en los lugares en los que se levantan los campos y ciudades que habitan los humanos, clasificándose, según diversos conjuntos, entre los entes; en toda esta realidad "al derecho", el contrasentido de las vastas empresas fallidas -en las que la política y la técnica desembocan en la negación de los proyectos que las presiden- manifiesta la inconsistencia del hombre, juguete de sus obras. (Levinas, 1998, p. 63, énfasis en el original).

De nuevo se oyen los ecos de Foucault acerca de la disolución del hombre; es más, Levinas reafirma el planteamiento foucaultiano: "La subjetividad, en tanto que disposición de estructuras inteligibles, no tendría

7 Véanse, por ejemplo, los ensayos de Günter Anders, recogidos en el primer volumen de La obsolescencia del hombre (1956). En el primero de los ensayos: "Sobre la vergüenza prometeica", Anders expone un párrafo titulado: "El hombre contemporáneo trata de escapar a esta calamidad mediante la asimilación física con los aparatos, mediante la Human Engineering. Perversión extrema de la oferta y la demanda" (Anders, 2011, p. 51); allí ilustra magistralmente el planteamiento levinasiano del hombre como "juguete de sus obras", en especial, en lo tocante a la técnica y cómo esta impacta en la nueva concepción (robotizada) de cuerpo (fisiotécnica), el cual tiene que responder a las exigencias de los nuevos aparatos, no al contrario: "No basta con interpretar el cuerpo; también hay que cambiarlo. Y renovarlo a diario y de forma diferente para cada aparato" (Anders, 2011, p. 54, énfasis en el original). 
ninguna finalidad interna. Asistiríamos a la ruina del mito del hombre" (1998, p. 67). No obstante, como se verá en el siguiente apartado, lo hará de un modo diferente al de Foucault: si bien Levinas coincide con diagnósticos de época (década de los sesenta) acerca tanto de la crisis de las humanidades como de la disolución de su objeto, su propuesta -aunque consciente de las situaciones contemporáneas que afectan radicalmente al sujeto y atenta a las nuevas subjetividades que brotan de ellas- no sucumbe ante la desesperanza que produciría la "ruina del mito del hombre", sino que, desde el rostro del otro -o mejor, desde su huella (trace)-, ofrece una posibilidad a ese rostro (visage) de arena amenazado de ser borrado por el mar.

\title{
Redecir lo humano
}

Una de las tensiones que encierra la obra de Levinas la constituye, sin duda, la relación entre ética y epistemología (o teoría del conocimiento). Es claro, sin embargo, que al postular la ética como filosofía primera, este autor revisa algunos postulados epistemológicos, sin necesariamente desembocar en irracionalidad. A este respecto dice Arthur Cools (2015):

\begin{abstract}
Levinas no define la racionalidad refiriéndose a un orden objetivo en el que ya estamos viviendo, ni fundamentando su argumento básico en la evidencia de una facultad subjetiva que nos es común; tampoco interpreta la racionalidad como la búsqueda del consenso, de un sentido o una verdad que nos unifique. Al contrario, una de las ideas básicas de su filosofía es la separación; es desde la perspectiva del ser separado, sin mediación, que él enfoca la cuestión de la racionalidad. No obstante, la filosofía de Levinas no desemboca en la irracionalidad. (p. 3).
\end{abstract}

Lo expresado por Cools resume, de modo preciso, tanto la postura epistemológica básica de Levinas como sus reflexiones en relación con las humanidades; por lo tanto, servirá de retícula para aproximarnos al texto que quizás condensa mejor la postura levinasiana sobre las humanidades: La significación y el sentido, escrito entre 1961 y 1963. El propósito de este apartado será mostrar cómo opera la racionalidad unívoca que ha seducido a las humanidades desde la modernidad, tornando obsoleto su lenguaje, y abrir un horizonte posible de reflexión para situarse por fuera de la dicotomía ciencias "duras"- ciencias humanas.

Si seguimos a Cools, habría tres fuentes posibles para justificar el conocimiento: la apelación al orden de los objetos, la apelación a un sujeto como dador de sentido y la apelación al consenso. En efecto, Levinas, en su ensayo, explora dichas fuentes. La primera la enuncia así: "Legítimamente, la realidad poseería desde el principio una significación. Realidad

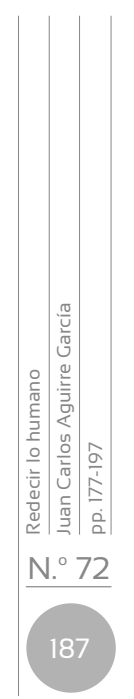


e inteligibilidad coincidirían. La identidad de las cosas comportaría la identidad de su significación" (1998, p. 20). La segunda la expresa de este modo:

De entrada, ningún dato estaría provisto de identidad ni podría penetrar en el pensamiento debido al efecto de un simple choque contra la pared de una receptividad. Darse a la conciencia, titilar para ella, exigiría que el dato, previamente, se situara en un horizonte iluminado (р. 22).

Finalmente, respecto al consenso, cuya manifestación externa sería el "objeto o gesto cultural", Levinas sostiene:

La acción cultural no expresa un pensamiento previo, sino, más bien, el ser al que dicha acción ya pertenece en tanto que encarnada. La significación no puede inventariarse en la interioridad de un pensamiento. El pensamiento mismo se inscribe en la Cultura a través del gesto verbal del cuerpo que lo precede y lo sobrepasa. La Cultura objetiva a la que, mediante la creación verbal, el pensamiento añade algo nuevo, lo ilumina y lo guía. (pp. 27-28, énfasis en el original).

Estas tres posturas han formado parte del panorama discursivo a lo largo de la historia del pensamiento, adoptando diferentes nombres y reformulándose de formas variadas. De igual manera, las tres han estado presentes en la configuración de las humanidades, bien sea en su pretensión naturalista o positivista, en su intento subjetivista por reivindicarse, o en la actual tendencia historicista, culturalista o cínica ${ }^{8}$. Para el caso específico de las humanidades, podría decirse que a Levinas le interesa destacar el desplazamiento que va desde la justificación basada en la intuición (sea empírica o eidética), pasando por la justificación basada en el contexto (de la cultura y del lenguaje) -esto es, la que utiliza la hermenéutica como método- y, a partir de estas, la justificación propia de la contemporaneidad en la que se establece una racionalidad basada en

8 El concepto de cinismo se define desde lo que Susan Haack (2007) Ilama nuevo cinismo (New Cynicism): "Quienes propusieron esta nueva cuasi ortodoxia, aunque difieren entre ellos en algunos detalles, fueron unánimes al insistir en que el supuesto ideal de investigación honesta, respeto por la evidencia, preocupación por la verdad, es un tipo de ilusión, una cortina de humo que disfraza las operaciones del poder, la política y la retórica [...] Apelar a los 'hechos' o a la 'evidencia' o a la 'racionalidad', afirmaban, no era sino sinsentido (hambug) que disfrazaba la exclusión de este o aquel grupo oprimido. La ciencia es, en su mayoría, un asunto de interés, negociación social o fabricación de mitos, la producción de inscripciones o narrativas; no solo no tiene una autoridad epistémica particular, ni un solo método racional, sino que realmente es, como toda 'investigación', solo política" (pp. 20-21). La razón de citar en extenso esta frase radica en que buena parte de la respuesta humanista ante la crisis de las humanidades enarbola argumentos que caerían en esta descripción; en consecuencia, dado que tales argumentos albergan seminalmente la irracionalidad, Levinas no puede alinearse con ellos. 
significaciones, a decir de Levinas, confusamente incestuosas, resultado de la "consanguinidad de la inteligencia con lo inteligible, enredados en la red del lenguaje, que nace en la expresión de la que sería inseparable el pensamiento" (Levinas, 1998, p. 32).

Aunque este desplazamiento también puede analizarse en las ciencias "duras", su presencia en las humanidades es relevante. En su última fase, las humanidades reciben con naturalidad la superación de la racionalidad dependiente del objeto (o del sujeto que recibe a ese objeto) y proclaman "la subordinación del intelecto a la expresión" (Levinas, 1998, p. 32). Tal subordinación requiere una "inversión del esquema gnoseológico" (p. 26), que sitúe el punto de partida ya no en el objeto o en un sujeto que piensa ese objeto, sino en "el espesor de la lengua y del mundo cultural pre-existentes" (p. 27). De este modo, ya no es la intuición sino la hermenéutica la llamada a develar el sentido.

La obsesión de las humanidades por develar el sentido (de una obra, un poema, un acontecimiento histórico), presupondría que hay un sentido por develar; no obstante, también es claro que la verdad que surgió en una perspectiva intelectualista (que defendía, por ejemplo, una versión correspondentista de la verdad), cedió su paso a una pluralidad de verdades dependientes de la cultura que, sin embargo, manifestaban sentidos de ser. Pareciera que este es el primer axioma de quien se ejercita en las humanidades, un axioma cuyo lema es la extinción de los hechos, con la única certeza de que solo hay interpretaciones. Ahora bien, independientemente de las discusiones que sobre este asunto se puedan generar ${ }^{9}$, la pluralidad de sentidos parece agotarse, tal y como lo han denunciado varios pensadores contemporáneos, en la "significación fija, privilegiada, que el mundo adquiere en función de las necesidades del hombre" (Levinas, 1998, p. 33) ${ }^{10}$. A esto lo llama Levinas: La significación "económi$\mathrm{ca}^{\prime \prime}$. Al respecto, afirma:

Se trataría de llevar la percepción a la ciencia que justifica la posible transformación del mundo; el hombre, a los complejos del psicoanálisis; la sociedad, a sus estructuras económicas. Sería necesario volver a encontrar por todas partes el sentido bajo la significación, la metáfora, la sublimación o la literatura. Habría, pues, significaciones "serias", reales, expresadas en términos científicos, orientadas por las

9 De hecho, en el propio terreno de la hermenéutica se está librando actualmente una batalla, similar a las guerras de las ciencias de mediados de los noventa, entre hermeneutas "realistas" y "anti-realistas". El líder de la postura realista es el hermeneuta italiano Maurizio Ferraris, quien ha propuesto un movimiento denominado Nouvo Realismo, en el que se confronta directamente la tesis de que "No hay hechos, solo interpretaciones" (véase Ferraris, 2013).

10 Entre los autores que, con claras huellas levinasianas, comparten este diagnóstico, pueden mencionarse a Bauman (2007) y Byung-Chul (2012). 
necesidades y, de manera general, por la economía. Solo la economía estaría verdaderamente orientada y sería significativa. Solo ella tendría el secreto de un sentido propio anterior al sentido figurado. (1998, p. 34).

Vuelven a oírse los ecos de posturas como la ilustrada por Illouz, citada al inicio de este artículo, con sus trágicas consecuencias: habría unas humanidades "serias" y otras que requieren pasar del sentido figurado al sentido real. Las primeras no estarían puestas en vilo; al contrario, serían bienvenidas en muchos contextos afines a los procesos productivos y, por ende, serían parte de cualquier proceso formativo, de todo plan de estudios. Las segundas tendrían la obligación de adaptarse a los "signos de los tiempos" o desaparecer.

Parece claro, entonces, que tanto las ciencias "duras" como las humanidades estarían orientadas por una "significación 'económica'" que acompasaría todos los sentidos posibles y los Ilamaría al orden. Justamente, es en este punto donde Levinas despliega su argumento: para que haya entendimiento entre los hombres, parece necesario que, al menos como ideal, haya un sentido que soporte la pluralidad de sentidos: “ $\mathrm{No}$ requieren las significaciones un sentido único del que toman prestado su propio significado?" (1998, p. 37). Sin embargo, esta univocidad no implica reducción del pluralismo:

[...] gracias a él [al ser, a la univocidad del ser], se elevan [las significaciones culturales] a la medida y a la esencia del ser, es decir, a su manera de ser. El ser no es de la manera como se cristaliza en esfera parmenídea, idéntica a sí misma, ni como criatura acabada y fija. (p. 35).

Ahora bien, la significación económica, que pretende ser ese sentido único, al establecer una línea divisoria entre lo "serio" y lo figurado, no parece salvaguardar las múltiples manifestaciones de sentido. Por tal motivo, concluye Levinas, "lo que hace falta es el sentido de los sentidos" (1998, p. 37). En lo que resta de este apartado, se intentará presentar esquemáticamente esta alternativa que, según la retícula adoptada de Cools, sería la idea levinasiana básica de separación.

El sentido económico - del que se acaba de sugerir que en vez de dar sentido, desorienta- responde a una perspectiva en la que se promueve una concepción de hombre y humanidad garante del pleno desarrollo de las capacidades del sujeto y respetuosa de sus derechos. No obstante, según Levinas, "el sentido es imposible a partir de un Yo (Moi) que existe, como dice Heidegger, de una manera tal que 'en su existencia le va la propia existencia'" (1998, p. 38). Lo paradójico es que, haciendo caso omiso de esta imposibilidad, las humanidades (aunque lo siguiente sea dicho por Levinas solo para la filosofía), "se produce[n] como una forma 
bajo la que se manifiesta el rechazo a comprometerse con lo Otro, la espera preferida a la acción, la indiferencia para con los otros" (p. 39). Esta obsesión por fundar lo humano en sentidos que broten del sujeto y su conciencia reflexiva es, precisamente, lo que conduce a la crisis de las humanidades (por no decir de todo el proyecto intelectual de Occidente) que es, en últimas, una "crisis de sentido" (p. 37).

De acuerdo con lo anterior, la respuesta a la crisis de sentido de las humanidades tendría que confrontar las perspectivas planteadas y renovar las fuentes de significación: “¿No indica el sentido, en tanto que orientación, un impulso, un fuera de sí hacia lo otro que uno mismo, mientras que la filosofía insiste en absorber todo Otro en el Mismo y en neutralizar la alteridad?" (Levinas, 1998, p. 39). A partir de esto puede considerarse una nueva dimensión de sentido, entendido ahora como la "orientación absoluta hacia lo Otro" (p. 41), que se respalda en las siguientes condiciones: (1) se trata de una salida de lo idéntico hacia otro absolutamente otro, (2) este movimiento del Mismo hacia lo otro, implica un no retorno, (3) la relación con lo Otro permite alcanzar al Otro, pero impide su reducción al Mismo. Estas condiciones serían los presupuestos tanto para responder a la crisis del sentido de las humanidades como para inaugurar el proyecto de redecir lo humano. Si bien cada una requeriría un desarrollo amplio, especialmente por los presupuestos filosóficos que controvierte y en los que se respalda, basta decir, para los propósitos de este artículo, que la adopción de esta nueva dimensión del sentido dotaría a las humanidades de la "juventud radical del impulso generoso" (Levinas, 1998, pp. 41-42), espíritu subsumido por Levinas bajo el término "liturgia": "El ejercicio de un oficio no solo totalmente gratuito, sino que requiere por parte de quien lo ejerce una inversión con pérdidas" (p. 42).

Ahora bien, las consecuencias que se desprenden de esta apuesta por redecir lo humano pueden focalizarse en dos campos: en primer lugar, en el terreno de la expresión; en segundo lugar, en el papel de las humanidades en el contexto de los saberes. En cuanto a lo primero, la expresión, sustento de la significación o inteligibilidad, no se reduciría a constatar lo existente (bien sea desde el naturalismo o desde el subjetivismo) o a celebrar el ser (desde la cultura o el lenguaje), sino que pasaría a ser

[...] una relación con aquel al que yo expreso la expresión y cuya presencia es ya requerida para que mi gesto cultural de expresión se produzca [...] Primordialmente, [el Otro] es sentido, en la medida en la que se lo otorga a la propia expresión, en la medida en la que solo por él un fenómeno como el de la significación se introduce, de suyo, en el ser. (Levinas, 1998, p. 45).

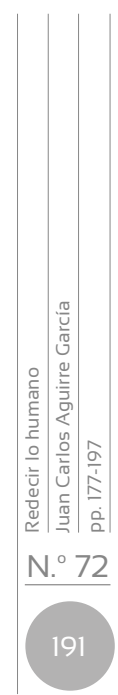


Según esta primera consecuencia de la tesis del redecir lo humano para las humanidades, es preciso situarse en una esfera discursiva previa, donde el privilegio no lo tenga la reflexión acerca de enunciados (sean lógicos o poéticos), sino la relación yo-Otro que los posibilita. El descentramiento del postulado clásico, según el cual las ciencias humanas son interpretativas (cuando no el sueño de igualarlas a los discursos cientificistas), consiste en reubicar el encuentro con el Otro como condición de cualquier enunciación. La expresión, por tanto, supera la tradicional concepción de ser una manera de acceder a lo oculto del hombre o la mujer, sino el modo como el Otro irrumpe la comodidad del mismo, le revela sentidos y lo interpela exigiéndole respuesta. En últimas, el redecir lo humano implica que la expresión se ancle en terrenos éticos (más que interpretativos o comprensivos), donde antes que comprender al Otro es necesario responderle, responder por él (lo que no implica renunciar a los intentos de comprensión).

En segundo lugar, en consonancia con la reconceptualización del rol de la expresión, las humanidades romperían la esfera cerrada de significaciones dependientes de los datos o de las comprensiones de las expresiones culturales (posibles detonantes de la crisis) y serían previas a esto; pero, además de ética, adquirirían una orientación crítica, capaz de juzgar la cultura, juzgar las civilizaciones: "La significación -lo inteligible- consiste para el ser en mostrarse en su simplicidad no histórica, en su desnudez absolutamente incalificable e irreductible, en existir 'antes' de la historia y 'antes' de la cultura" (Levinas, 1998, p. 53). Por tanto, un rol primordial de las humanidades, antes de preocuparse por la esencia del sujeto, sus derechos, su modos de comportarse, su Mismidad, tendrá que orientarse, como se ha dicho, por el Otro absoluto, separado; es el Otro quien determina los modos de aproximación y, ante todo, exige una respuesta. Ahora bien, esta respuesta inaugura una esfera de justicia, en la que se vuelve central la posibilidad de poner en cuestión aquellos discursos que encubran los intentos por deshacer la alteridad del Otro, reduciéndolo a esquemas de mismidad. En síntesis, gracias a esta segunda consecuencia de la tesis del redecir lo humano, se desplaza el interés tematizante y se le da prioridad a la responsividad ética que implica, a su vez, denunciar, a partir del criterio del Otro como absolutamente Otro, las injusticias que se cometen y actuar según su demanda, sin importar las razones que haya (aún si no las hubiera).

Este apartado permitió considerar cómo las humanidades se han trenzado en modos de pensar su objeto que coinciden con una racionalidad aparentemente multiforme pero que, en últimas, guarda la pretensión de comprenderlo todo. Según el análisis de Levinas, esta sería la raíz de su crisis. La propuesta del autor, condensada aquí bajo la 
expresión redecir lo humano, consiste en explorar las fuentes de sentido previas a estas fuentes privilegiadas por la racionalidad occidental. Antes que de un sujeto autárquico que dirige su mirada y da sentido, el sentido brotaría de una exterioridad, de un más allá, de una altura. Por tanto, el Otro irrumpe la comodidad del Mismo exigiéndole una respuesta. La nueva dimensión de sentido permitiría a las humanidades romper los esquemas que las comprometen con un proyecto fijo de racionalidad, lanzándolas a terrenos poco seguros. Es por ello que la expresión "juventud radical del impulso generoso" podría servir de lema para las humanidades por venir: si bien las condiciones actuales para el desarrollo de las humanidades parecen presagiar su extinción (al menos en las estructuras en las que tradicionalmente se han cultivado), en la medida en que tengan como obsesión la responsabilidad por el Otro y la seguridad de que de ella brota su sentido, los humanistas de hoy podrán repetir las palabras de León Blum, quien en pleno horror nazi exclamaba: "trabajamos en el presente, no para el presente" (citado por Levinas, 1998, p. 42).

\section{Conclusión}

Sin lugar a dudas, la crisis de las humanidades se refleja, entre otros síntomas, en el intento por desaparecer programas académicos que las cultivan o su adscripción a departamentos o facultades en los que pueden restringirse o difuminarse sus intereses investigativos; asimismo, un indicio de la crisis es la reducción de fondos para investigaciones en humanidades o en la seducción para que tales investigaciones respondan a intereses gubernamentales. También puede notarse en la reducción de estudiantes de posgrado dedicados a las humanidades y la poca oferta académica al respecto (hablo de las humanidades como corpus; es claro que, por separado, proliferan programas de posgrado en disciplinas humanísticas, mas no abundan los posgrados en humanidades). El que no haya oferta ni demanda de posgrados en humanidades, aunque haya muchos posgrados dedicados a disciplinas humanísticas, podría contribuir a la dispersión de esfuerzos investigativos, que se centran en asuntos demarcados o especializados de una disciplina particular, obviando discusiones de fondo sobre, por ejemplo, el sentido de las humanidades, limitándose a aclarar o abordar los fundamentos de una disciplina particular.

Además de la demanda, por ejemplo en concursos docentes, de expertos en disciplinas consolidadas de las ciencias humanas (filosofía, antropología, historia, literatura), existe la incertidumbre acerca de cuál 
sería el perfil laboral o profesional de una persona con título de posgrado en humanidades o ciencias humanas. Igualmente, se torna difícil, ante la amplitud de problemáticas que abordan las humanidades, elegir un conjunto de problemas relevantes que se incluyan en un plan de estudios de tal posgrado. Pareciera haber una anarquía que por lo general se subsana con los intereses políticos predominantes de un grupo de docentes o unos grupos de investigación.

Si bien en nuestro contexto la formación universitaria es el nicho privilegiado para el cultivo de las disciplinas humanísticas, parece que la situación, antes que promisoria, estaría contribuyendo a radicalizar la crisis de las humanidades. No obstante, la propuesta de Levinas, aquí considerada, podría revaluar el sentido de la formación en humanidades a partir de una perspectiva sensible a las demandas del Otro. Esto exigiría un conocimiento radical de los paradigmas que han guiado la exploración sobre lo humano, una crítica atenta a las dificultades de los discursos humanistas -tanto de los que reducen lo humano a ideales naturalistas como los que cohonestan con posturas relativistas, escépticas, nihilistas o cínicas-, una alteración del criterio de la razón adulta y un aventurarse, con impulso juvenil y generoso, hacia lo desconocido, lo incierto, lo inseguro, lo exterior y, especialmente, orientándose por el Otro como absolutamente Otro.

Las razones para la vigencia de las humanidades, en ocasiones soportadas en frases rimbombantes -por no decir falaces- como: la necesidad de que el humano sea auténticamente humano, o que sin ellas los hombres se convertirían en máquinas o autómatas, o que con ellas se forman mejores ciudadanos, $\mathrm{u}$ otras por el estilo ${ }^{11}$, solo pueden adquirir un sentido pleno cuando asuman la radicalidad de su compromiso de responder al Otro, denuncien las situaciones de injusticia ante todo Otro y encuentren medios de expresión originales que, sin renunciar a la racionalidad, permitan que lo humano sea dicho y celebren la excedencia

11 En este mismo sentido, la profesora Claire Elise Katz afirma: "la idea que el estudio de la filosofía, o incluso de las humanidades en general, nos haría mejores personas, circula por todas partes en la academia [...] Solo tenemos que ir a Kant o a las interpretaciones sobre Kant para ver cómo la idea que estudiar filosofía, entender lo que es una acción correcta y luego elegir qué hacer en esas situaciones se iguala y se reduce a meros procedimientos de toma de decisiones [...] Entre otras creencias erróneas, esta idea asume que hay una cosa correcta, que nuestras obligaciones están claramente definidas, que la ética no es complicada y que mis obligaciones éticas se parecen a una 'lista' de cosas por hacer" (2013, p. 5). El estudio de la profesora Katz es un claro ejemplo de cómo la filosofía de Levinas puede contribuir a la "solución" de la crisis de las humanidades a partir de un "nuevo humanismo" "basado en una subjetividad que se define cediendo el estatus egocéntrico por/para el otro" (p. 6). Lo interesante de su análisis es que se dedica, sobre todo, a contextualizar la discusión en el ámbito educativo. 
de lo humano no reductible a significaciones unívocas ${ }^{12}$. En consecuencia, la vigencia de las humanidades está inexorablemente unida a su posibilidad de, constantemente, redecir lo humano.

12 Esto, obviamente, exige un replanteamiento del concepto de teoría, tan querido en la sociedad de la información. A este respecto dice Byung (2014): "Sin la seducción del otro atópico, que desata en el pensamiento un deseo erótico, aquel que se atrofia y no pasa de ser un mero trabajo, que reproduce siempre lo mismo. Al pensamiento calculador le falta la negatividad de la atopía. Este es trabajo en lo positivo. Ninguna negatividad le provoca inquietud [...] El pensamiento se hace 'más fuerte', 'más inquietante' en el momento en que, tocado por el aletazo del Eros, intenta llevar al lenguaje al otro atópico, carente de lenguaje. Al pensamiento calculador, guiado por los datos, le falta la resistencia del otro atópico. El pensamiento sin Eros es meramente repetitivo y aditivo. $\mathrm{Y}$ el amor sin Eros, sin su fuerza ascensional, degenera hasta la 'mera sensibilidad'. Sensibilidad y trabajo pertenecen al mismo orden. Carecen de espíritu y deseo" (p. 37). 


\section{Referencias}

Anders, G. (2011). La obsolescencia del hombre. Volumen I: Sobre el alma en la época de la segunda revolución industrial. Valencia: Pre-textos.

Bauman, Z. (2007). Vida de consumo. México: Fondo de Cultura Económica.

Blumenberg, H. (2013). Historia del espíritu de la técnica. Valencia: Pre-textos.

Byung-Chul, H. (2012). La sociedad del cansancio. Barcelona: Herder.

Castro, R. (2005). La frase de Foucault: "El hombre ha muerto". Alpha, 21, 225-233. doi: 10.4067/S0718-22012005000100015

Cederberg, C. (2010). Resaying the Human. Levinas Beyond Humanism and Antihumanism. Estocolmo: Södertörns Högskola.

Cools, A. (2015). Levinas' defense of intellectualism: An undecidable ambiguity? En A. Breitling, C. Bremmers \& A. Cools (eds.) Debating Levinas' Legacy (pp. 3-15). Leiden / Boston: Brill.

Cuartango, P. (2014, 8 de junio). La crisis de las humanidades. El mundo. Recuperado de: http://www.elmundo.es/opinion/2014/06/08/ 5394b1a4268e3ed52b8b4580.html

Delbanco, A. (2013, 23 de noviembre). La crisis de las humanidades. La nación. Recuperado de: http://www.nacion.com/opinion/foros/ crisis-humanidades_0_1380061988.html.

Foucault, M. (2001). Las palabras y las cosas. Una arqueología de las ciencias humanas (30 ed). México: Siglo xxI.

Greteman, B. (2014, 13 de junio). It's the End of the Humanities as We Know It. And I Feel Fine. New Republic. Recuperado de: https:// newrepublic.com/article/118139/crisis-humanities-has-long-history.

Gutting, G. (2013, 30 de noviembre). The Real Humanities Crisis. The New York Times. Recuperado de: http://opinionator.blogs.nytimes. com/2013/11/30/the-real-humanities-crisis/.

Haack, S. (2002). The same, only different. Journal of Aesthetic Education, 36 (3), 34-39.

Haack, S. (2007). Defending science-within reason. Nueva York: Prometheus.

Illouz, E. (2007). Intimidades congeladas. Las emociones en el capitalismo. Buenos Aires: Katz.

Illouz, E. (2012). Why love hurts? A sociological explanation. Cambridge: Polity Press.

Jiménez, M. (2014, 23 de junio). Las humanidades, cada vez más cerca de su fin: el STEM acabará muy pronto con ellas. El confidencial. Recuperado de: http://www.elconfidencial.com/alma-corazon-vi- 
da/2014-06-23/las-humanidades-Ilegan-a-su-fin-el-stem-esta-acabando-con-ellas_149888/

Katz, C. (2013). Levinas and the crisis of humanism. Bloomington: Indiana Univesity Press.

Levinas, E. (1998). Humanismo del otro hombre. Madrid: Caparrós.

Nussbaum, M. (2010). Sin fines de lucro. Por qué la democracia necesita de las humanidades. Buenos Aires: Katz.

¿Quién piensa en las artes y las humanidades? (2016, 9 de julio). Recuperado de: http://www.semana.com/cultura/articulo/andres-oppenheimer-las-ciencias-sociales-y-el-arte/481148.

Reisz, M. (9 de Julio de 2015). Humanities crisis? What crisis? Times Higher Education. Recuperado de: https://www.timeshighereducation. com/news/humanities-crisis-what-crisis

Sibilia, P. (2005). El hombre postorgánico. Cuerpo, subjetividad y tecnologías digitales. Buenos Aires: Fondo de Cultura Económica.

Vargas, G. \& Prada, M. (2016, mayo). Convocatoria 72. La puesta en vilo de las humanidades. Recuperado de: http://revistas.pedagogica.edu. co/index.php/RCE/announcement/view/76

Wallerstein, I. (2006). Abrir las ciencias sociales (9 ed.). México: Siglo xxı.

Wolfe, C. (2010). What is posthumanism? Londres: University of Minnesota Press. 